\title{
Strengthening Mechanisms and Dislocation Dynamics in Twinned Metal Nanowires
}

\author{
A.J. Cao, Y.G. Wei, and Scott X. Mao
}

Microtwins are frequently observed in face-centered-cubic (fcc) metal nanowires with low stacking fault energy. The authors have previously reported that the tensile yield strength of copper nanowires can be increased by the presence of twin boundaries. In this work simulations are carried out under both uniaxial tension and compression loading, to demonstrate that the strengthening effects are inherent to these nanowires, independent of the loading condition (tensile/compressive). It appears that the strengthening mechanism of the twinned nanowires can be attributed to stress redistribution due to the change of crystallographic orientations across twin boundaries, which requires larger external stress to make them yield as compared to the twin-free wire.

\section{INTRODUCTION}

As one of the most important onedimensional nanostructures, nanowires have attracted a great deal of interest due to their pivotal role in future electronic, optoelectronic, and nano-electromechanical systems. ${ }^{1,2}$ The structure and physical properties of nanowires can be quite different from those of bulk materials due to the effects of larger free surfaces. For example, Y. Kondo and K. Takayanagi ${ }^{3}$ observed that a face-centered-cubic (fcc) $\{100\}$ oriented gold film changed to an fcc $\{111\}$ oriented film at film thickness of less than eight atomic layers. When the $<110>$ gold nanowire is thinned below $1.5 \mathrm{~nm}$ in diameter, it forms a helical multi-shell structure composed of coaxial tubes. ${ }^{1}$ More recently, W. Liang et al. ${ }^{4,5}$ and H.S. Park et al. ${ }^{6}$ have shown that some fcc single-crystalline metal wires, such as copper, nickel, and gold, exhibit pseudo-elasticity and shape

memory effects when the wire size is below $5 \mathrm{~nm}$.

Meanwhile, experimentally, twins, a class of planar defects, are observed most often in metal (copper, silver, and gold) nanowires with [111] growth orientation. ${ }^{7,8}$ Recently, twinned structures have also been reported in other semiconductor and ceramic materials, such as $\mathrm{GaP},{ }^{9} \mathrm{ZnS},{ }^{10}$ and $\mathrm{SiC} .{ }^{11}$ Because twin boundaries (TBs) in these nanowires will strongly affect their mechanical and physical properties, it is of great significance to investigate the details

\section{$=$}

$\$$

How would you...

...describe the overall significance of this paper?

We demonstrate that the microstructure strengthening

- effects are still valid in nanoscale materials, which can be used as potential strategies to design materials with these structures.

...describe this work to a materials science and engineering professional with no experience in your technical specialty?

This work highlights that the strengthening mechanism of the twinned nanowires is due to the stress redistribution along the wire axis, requiring larger external stress to make them yield as compared to twin-free wire. The simulation results show that twin boundaries mainly serve as a barrier for dislocation movements, which contribute to some

hardening effects.

...describe this work to a layperson?

Twinned metal nanowire has different mechanical behavior than bulk metals. This study demonstrates that the mechanical properties of metal nanowires can be controlled by tailoring interior growth twin structures. of twin structures and their roles in the nanowires. ${ }^{12,13}$ This work focuses on revealing the strengthening mechanisms and dislocation dynamics due to twinning in copper nanowires.

\section{VISUALIZING DEFECTS IN NANOWIRES}

As a model, this study focuses on twinned copper nanowires with $<111>$ orientations and nearly square cross sections, as indicated by experimental observations. ${ }^{7,8}$ The initial configuration is constructed by repeating $\Sigma 3$ coherent twins in the $<111>$ axis orientation. ${ }^{14}$ Five samples are prepared for simulations. All the twinned nanowires consist of equally spaced twins. In this work, the twin boundary spacing (TBS) of four twinned wires is chosen as $16 \mathrm{~nm}, 10.6 \mathrm{~nm}, 8 \mathrm{~nm}$, and $6.4 \mathrm{~nm}$, respectively. For comparison, a $<111>$ twin-free nanowire with ( $\overline{1} \overline{1} 2)$ and (1 $\overline{1} 0)$ lateral surfaces is also prepared. The initial configurations of the three twinned samples are shown in Figure 1. The sizes of the wires are the same for all these nanowires, which are $8 \mathrm{~nm} \times 8$ $\mathrm{nm} \times 32 \mathrm{~nm}^{3}$. Up to 175,000 atoms are contained in the simulation model. An embedded-atom method (EAM) potential of Y. Mishin et al. ${ }^{15}$ is chosen in the present simulations because it is calibrated according to the ab initio calculated values of stacking fault and twin formation energies. ${ }^{15}$

To visualize defects in the nanowires, colors are assigned to the atoms according to a local crystallinity classification visualized by common neighbor analysis ${ }^{16}$ that permits the distinction between atoms in a local hexagonal-close-packed (hcp) environment and those in an fcc environment. A single line of hcp atoms represents a twin boundary, two adjacent hcp lines stand 
for an intrinsic stacking fault, and two hcp lines with an fcc line stand for an extrinsic stacking fault between them. Other twelve coordinated atoms and non-twelve coordinated atoms appear in the free surface region and in the core of dislocations. This is the same method used in References 17 and 18.

After the initial construction, a conjugate gradient method is used to minimize the whole wire energy in order to obtain a more stable configuration. Then the samples are thermally equilibrated to $300 \mathrm{~K}$ for 20 picoseconds (ps) using a Nosé-Hoover thermostat. ${ }^{19,20}$ Free boundary conditions are utilized in all three dimensions in the whole work.

Uniaxial tensile and compressive loading is applied under the simulated quasistatic conditions. ${ }^{5,17}$ To accelerate simulation efficiency, in the first several loading steps all the atoms are first displaced in a prescribed uniform strain increment of $0.5 \%$ ( $-0.5 \%$ in compression) in the length direction, and $0.2 \%$ $(-0.2 \%$ in compression) strain increment is applied in the remaining steps. The wires are then relaxed at a constant temperature of $300 \mathrm{~K}$ for $25 \mathrm{ps}$ at their terminals to obtain a macroscopic equilibrium configuration. The equilibrated process usually takes less than $15 \mathrm{ps}$, and the average stress over the last 5 ps of the relaxation period is taken as the stress of the nanowire. The stress reported in this work is calculated using the virial theorem, which is equivalent to the Cauchy stress in the average sense. ${ }^{21}$

A typical stress-strain curve of the wire with five twins is shown in Figure 2a. The compressive yield stress as a function of TBS for the simulated wires is shown in Figure 2b. In Figure 2a, the wires show a linear increase in stress with increasing strain in the elastic deformation stage. The Young's modulus calculated from the stress-strain curve is about $200 \mathrm{GPa}$, consistent with experimental results. After the elastic deformation, the stress-strain curve shows a precipitous drop, implying that yielding has occurred. Clearly, one can see from Figure $2 b$ that there is an increase in yield stress associated with the twinned sample as compared to that of twin-free wire. It is important to note that there exists a clear correlation between strengthening effects and twin sizes in the nanowires (i.e., the smaller the TBS, the higher the yield stress). Note that the twin strengthening effects have also been found in gold nanopillars by other researchers. ${ }^{22}$

To address the strengthening mechanism caused by TBs, the distribution of the normal component $\left(\pi^{33}\right)$ of the atomic level stress ${ }^{17}$ on part of a section along the wire axis is shown in Figure 3. Due to the large surface-to-volume ratio, nanowires sustain high interior

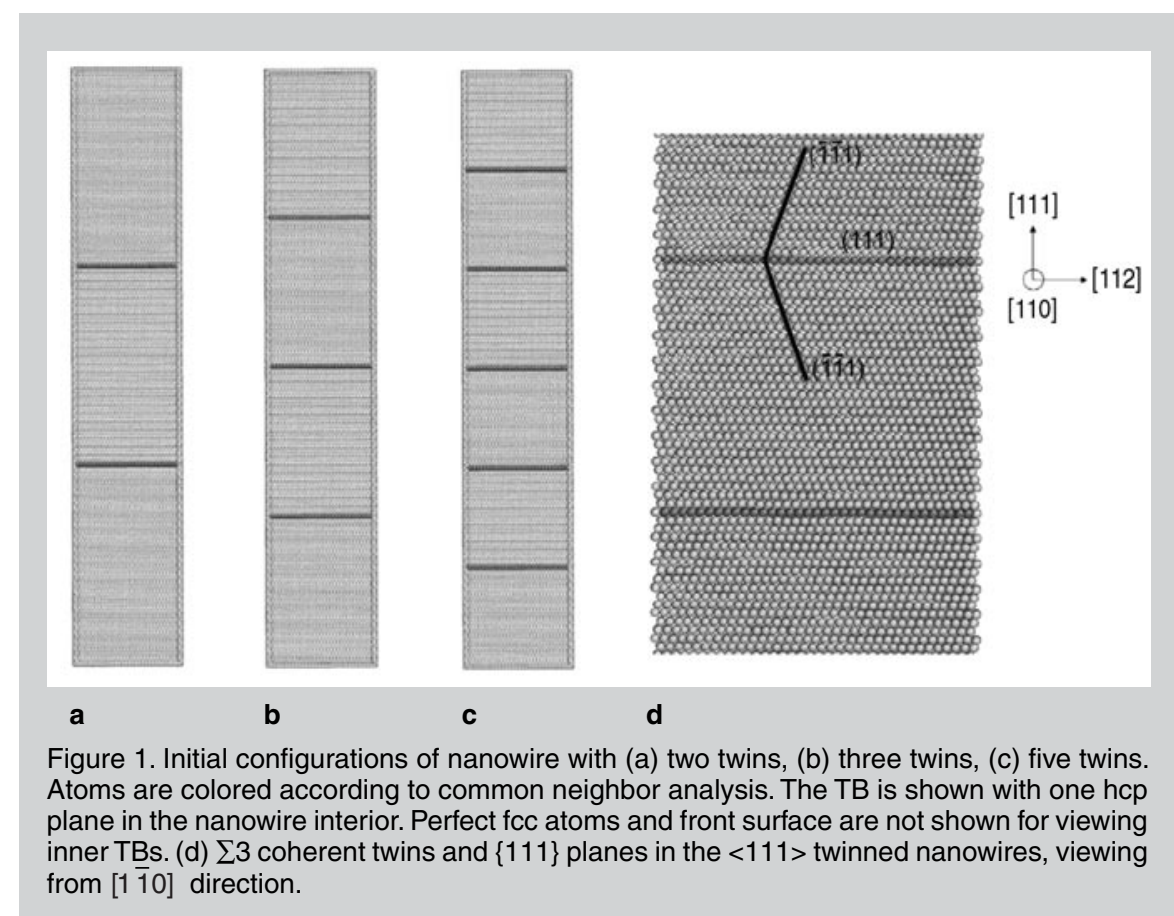

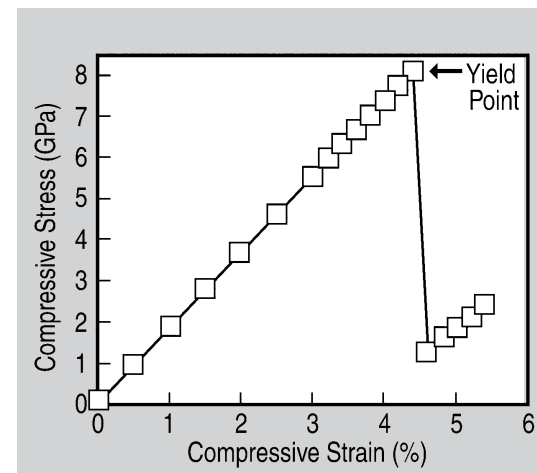

a

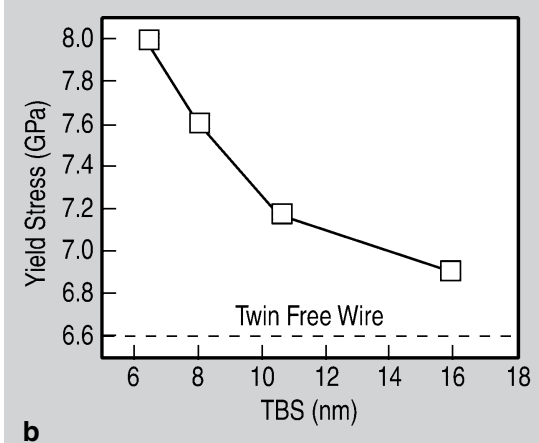

Figure 2. (a) A typical compressive stressstrain curve for the copper nanowires containing five twins. (b) Compressive yield stress as a function of TBS for the simulated nanowires.

compressive stresses induced by tensile surface stresses. In the twin-free wire (Figure 3a), the stress is quite uniform in the interior at equilibrium state ( $\mathrm{T}=0 \mathrm{~K}$ after energy minimization). In the case of twinned nanowire (Figure $3 b$ ), however, the stress around the TBs varies in a heterogeneous way and the difference between the interior compressive stress and the tensile surface stress is smaller than that of twin-free wire. The places near the intersections of twin boundary and free surfaces play an important role in determining the yield stress (to be discussed latter). Clearly, redistribution of atomic-level stress is due to the presence of TBs. Because of the anisotropic nature of crystals, the change of crystallographic orientation in twin and matrix cause their structure and mechanical properties variation across TBs. In this sense, the TB strengthening effect is reminiscent of the common route using a grain boundary refinement mechanism in bulk conventional metals.

\section{STREGTHENING EFFECTS OF LOADING CONDITIONS}

It is generally accepted that yielding 

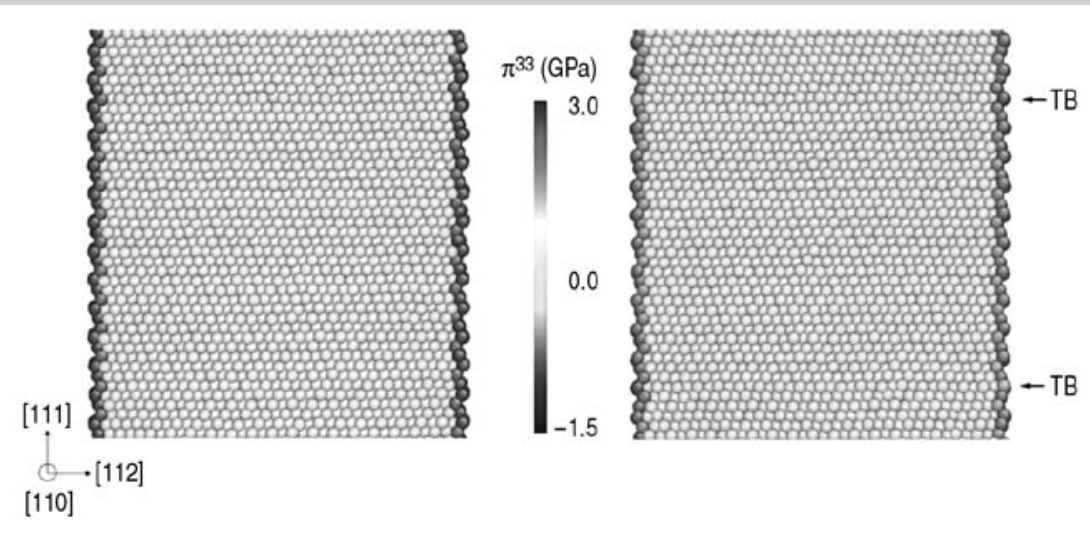

a

b

Figure 3. The distribution of atomic-level normal stress tensor component, $\pi^{33}$, on the section along the wire axis in the (a) equilibrated twin-free copper nanowire and (b) equilibrated five-twin copper nanowire.

of nanowires is caused by both the externally applied forces and the surfaceinduced interior stresses. ${ }^{12,13}$ The yield criterion, however, is still a topic of considerable interest to interpret the observed yielding behavior in nanowires. It appears that the local stress (or gradient) near free surfaces is more important than the wire interior average stress in controlling the yielding behavior of metal nanowires since the first dislocation comes out from the surface edges of the wires.

The resolved shear stress (RSS) has been examined based on the calculated atomic level stress. It turns out that the twinned wires do exhibit smaller RSS on certain $\{111\}$ slip planes near the intersection of TBs and free surfaces because of the above-mentioned stress redistribution scenario. As a result, a larger external stress is required to make the wire yield, assuming a constant critical resolved shear stress for wires of the same size. For example, the near-surface RSS on the observed slip plane of the equilibrated wire with five twins is $0.24 \mathrm{GPa}$, much smaller than that of the twin-free one, $0.45 \mathrm{GPa}$. As such, a trend of decreasing value of RSS near the surface with decreasing TBS is observed, which is consistent with an increase in yield strength with decreasing TBS.

It is expected that the local RSS is opposite in sign for the same $\{111\}$ slip plane in the [111] wires when subjected to tensile and compressive loading. The external force has the same positive contribution to the local RSS during loading. In other words, external tensile and compressive forces both increase the magnitude of RSS, so one can anticipate that both tensile and compres- sive loading should lead to the same size-dependant yield stress trends. This is what simulations have captured in this study. Based on the above reasoning, the strengthening effects do not change due to different loading condition (tension/compression) and the local RSS near-surface region is a reasonable yielding criterion for interpreting the twin size dependence of the yield stress phenomenon. Note that this scenario is also valid for the case of wire size dependence of yield stress. ${ }^{23}$

\section{DISLOCATION DYNAMICS DURING MECHANICAL STRAINING}

Dislocation dynamics during mechanical straining was also monitored and analyzed. Upon tensile loading of the $\langle 111>$ twinned nanowires, the first leading partial dislocation with Burgers vector of $1 / 6<112>$ is nucleated from

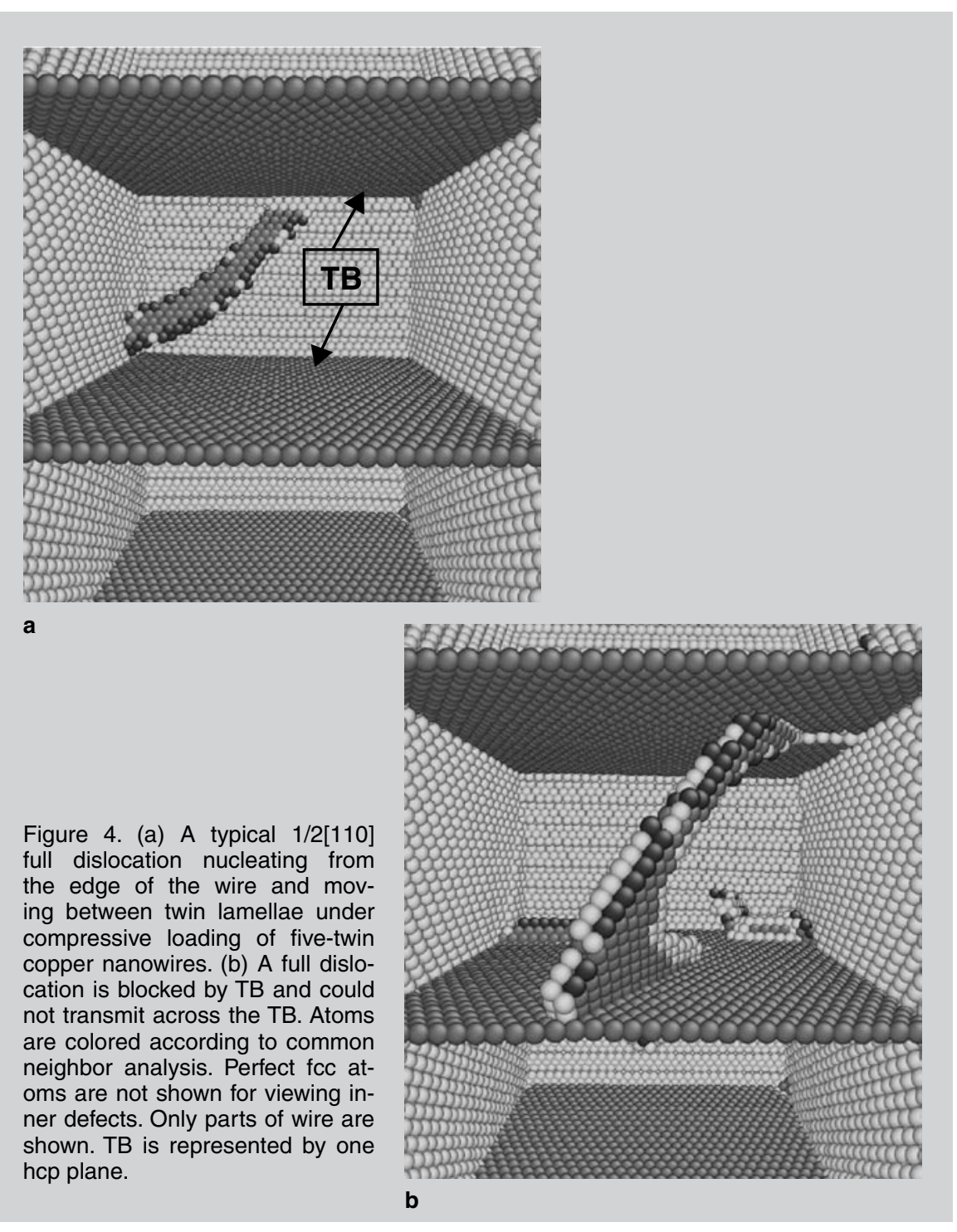


the edge of the free surface, and with further straining the movement of dislocation is blocked by TBs. ${ }^{12,18}$ In compression, the observed dislocations are all in 1/2[110] type. A typical snapshot of $1 / 2$ [110] full dislocations is shown in Figure $4 \mathrm{a}$. Figure $4 \mathrm{~b}$ shows that the trapped dislocation cannot egress out of the wire and leave on the TBs. While in the twin-free wire, dislocations glide freely across the whole wire and get incorporated in the opposite site of the side surfaces and no dislocations are left in the wire.

The different dislocation behavior involved in tension and compression can be fully understood from a Schmid factor analysis. In compression, slip of full dislocations would be crystallographically favored compared to partial slip and twinning, because the Schmid factor for a trailing partial (dislocation) is higher $(0.31)$ than the leading one (0.16) in a $<111>$-oriented pillar. The trailing partial, therefore, almost simultaneously follows the leading one and as such creates the observed full dislocation. On the contrary, for tensile loading, the Schmid factor of the trailing partial (0.16) is smaller than that for the leading one (0.31). Thus the trailing partial would not immediately follow, emanating from the same nucleation site, such that the leading partial leaves a stacking fault behind. ${ }^{24}$

Another intriguing finding is that ductility (tensile elongation) of the twinned wires is not notably reduced by the TB interfaces (Figure 2a in Reference 18). Note that the dislocation dynamics and fracture scenario revealed here are different than those in the wire containing bamboo-like grain boundary structures, ${ }^{25}$ in which the high-angle grain boundaries reduce the yield strength and ductility. The perfect coherent TB structure with low interface energy results in less propensity for necking instability, endowing the nanowires with both high strength and good plastic deformation capability, which make them attractive for structural applications.

\section{CONCLUSION}

Simulation results show that TBs play a vital role in controlling the plastic deformation and fracture mechanisms of nanowires. The simulations have shown that higher yield strength is inherent to the twinned copper nanowires, as compared to that of the twinfree wire, independent of loading condition. Twin boundary strengthening mechanisms can be attributed to the redistribution of atomic-level stresses due to the presence of TBs. This study demonstrates that the mechanical properties of metal nanowires can be controlled by tailoring interior twin structures. The twinned nanowires may have a wide range of applications in nanodevices and nanoelectric mechanical systems because of their high strength and good inelastic deformation capability.

\section{References}

1. Y. Kondo and K. Takayanagi, Science, 289 (2000), p. 606.

2. S. lijima et al., Science, 296 (2002), p. 611.

3. Y. Kondo and K. Takayanagi, Phys. Rev. Lett., 79 (1997), p. 3455.

4. W. Liang, M. Zhou, and F. Ke, Nano Lett., 5 (2005), p. 2039.

5. W. Liang and M. Zhou, Phys. Rev. B, 73 (2006), no. 115409.

6. H.S. Park, K. Gall, and J.A. Zimmerman, Phys. Rev. Lett., 95 (2005), no. 255504.

7. M.L. Tian et al., Nano Lett., 3 (2003), p. 919.

8. J.G. Wang et al., J. Phys. Chem. B, 108 (2004), p. 841.

9. Q. Xiong, J. Wang, and P. Eklund, Nano Lett., 6 (2006), p. 2736.

10. Y. Hao et al., Nano Lett., 6 (2006), p. 1650.

11. D. Wang et al., Nanotechnology, 19 (2008), no. 215602.

12. A.J. Cao and Y.G. Wei, Appl. Phys. Lett., 89 (2006), no. 041919 .

13. A.J. Cao and Y.G. Wei, J. Appl. Phys., 102 (2007), no. 083511 .

14. J.M. Howe, Interfaces in Materials (New York: Wiley, 1997).

15. Y. Mishin et al., Phys. Rev. B, 63 (2001), no. 224106.

16. J.D. Honeycutt and H.C. Andersen, J. Phys. Chem., 91 (1987), p. 4950.

17. A.J. Cao and Y.G. Wei, Phys. Rev. B, 74 (2006), no. 214108.

18. A.J. Cao, Y.G. Wei, and S.X. Mao, Appl. Phys. Lett., 90 (2007), no. 151909.

19. S. Nosé, J. Chem. Phys., 81 (1984), p. 511.

20.W.G. Hoover, Phys. Rev. A, 31 (1985), p. 1695.

21. K.S. Cheung and S. Yip, J. Appl. Phys., 70 (1991), p. 5688.

22. K.A. Afanasyev and F. Sansoz, Nano Lett., 7 (2007), p. 5056.

23. A. Cao, Y. Wei, and S.X. Mao, Scripta Mater., 59 (2008), p. 219.

24. H. Fang et al., Computer Methods in Applied Mechanics and Engineering, 193 (2004), p. 1789.

25. A. Cao, Y. Wei, and E. Ma, Phys. Rev. B, 77 (2008), no. 195429.

A.J. Cao (graduate student) and Y.G. Wei (professor) are with the State Key Laboratory of Nonlinear Mechanics, Institute of Mechanics, Chinese Academy of Sciences, Beijing 100080, P.R. China. Scott X. Mao (William Kepler Whiteford Professor) is with the Department of Mechanical Engineering and Materials Science, University of Pittsburgh, Pittsburgh, PA 15261, and can be reached at smao@ngr .pitt.edu. Dr. Mao is also Guangbiao professor in the Department of Materials Science and Engineering, Zhenjiang University, China. 